Artigo Original

\title{
Gasto energético e consumo calórico em adolescentes do sexo masculino com diferentes níveis de adiposidade corporal
}

\author{
Diego Augusto Santos Silva \\ Rosane Carla Rosendo da Silva \\ Edio Luiz Petroski
}

\author{
Programa de Pós Graduação em Educação Física, Centro de Desportos, \\ Universidade Federal de Santa Catarina, Florianópolis, SC, Brasil
}

\begin{abstract}
Resumo: Foram comparados o gasto energético e consumo calórico de adolescentes com diferentes níveis de gordura corporal. Estudo transversal com 40 adolescentes de 14 a 16 anos do sexo masculino. Os adolescentes foram divididos em dois grupos, cada um com 20 sujeitos, de acordo com o \%G: baixo (\%G < $11 \%$ ) e alto ( $\% G>20 \%)$. O gasto energético foi mensurado por acelerômetros triaxiais e recordatório de atividades diárias. $\mathrm{O}$ consumo calórico foi avaliado pelo registro de 24 horas. Os jovens com baixo \%G tiveram menor consumo calórico $\left(\bar{X}=2408,1 \pm 557,9 \mathrm{kcal}^{-1 a^{-1}}\right)$ em comparação aos jovens com alto \%G $(\bar{X}$ $\left.=2931,7 \pm 679,2 \mathrm{kcal} \cdot \mathrm{dia}^{-1}, \mathrm{p}<0,05\right)$. Ademais, apresentaram gasto energético relativo maior em comparação aos jovens com alto \%G. Adolescentes com \%G acima do ideal consomem mais calorias do que gastam, sendo o inverso verdadeiro para aqueles com baixa adiposidade corporal.
\end{abstract}

Palavras-chave: Antropometria. Obesidade. Atividade física. Comportamento alimentar.

\section{Energy expenditure and caloric intake among male adolescents with different levels of body adiposy}

\begin{abstract}
Energy expenditure and caloric intake were compared among adolescents with different levels of adiposity. A cross-sectional study was carried with 40 adolescent boys aged 14 to 16 years. The adolescents were divided into two groups of 20 subjects each, according to \% body fat (\%BF): low (<11\%) and high (> $20 \%$ ). Energy expenditure was measured using accelerometer and daily activity diary. The dietary pattern was evaluated based on 24-h recall. Caloric intake was lower in adolescents with low \%F ( $\bar{X}=2408.1 \pm$ 557.9 kcal.day $\left.^{-1}\right)$ compared to those with high $\% \mathrm{~F}\left(\bar{X}=2931.7 \pm 679.2\right.$ kcal.day $\left.^{-1}, \mathrm{p}<0.05\right)$. In addition, they presented greater relative energy expenditure than adolescents with high $\% \mathrm{~F}(\mathrm{p}<0.05)$. High $\% \mathrm{~F}$ adolescents showed larger caloric intake than expenditure, while the opposite was observed among low \%F males.
\end{abstract}

Keywords: Anthropometry. Obesity. Physical activity. Food behavior.

\section{Introdução}

O sobrepeso e a obesidade durante a adolescência são considerados importantes problemas de saúde pública em países desenvolvidos e em desenvolvimento. $\mathrm{O}$ acúmulo excessivo de gordura corporal está associado ao aumento no risco de desenvolver doenças crônicas em idades precoces, como a hipertensão e diabetes (BAKER et al., 2007). Adolescentes acima do peso, especialmente quando apresentam excesso de gordura na região do tronco, estão mais propensos a apresentarem um conjunto de anormalidades, conhecida como síndrome metabólica (LEITE et al., 2009).

Além do sobrepeso e obesidade, países em desenvolvimento, como o Brasil, ainda são afetados, por situações de baixo peso e desnutrição energético-proteica (MARTINS; SAWAYA, 2006). Tais situações aumentam a probabilidade de déficits nutricionais e problemas relacionados ao crescimento físico (MARTINS; SAWAYA, 2006; SANTOS et al., 2009).

A composição corporal é influenciada por múltiplos fatores, passando desde aspectos genéticos, metabólicos e fisiológicos a situações relacionadas ao estilo de vida como, por exemplo, prática de atividade física e hábitos alimentares (DAYRELL et al., 2009). Eisenmann (2006), em sua revisão de literatura sobre a tendência secular da obesidade entre jovens, apontou como principais causas a diminuição da atividade física e o aumento da ingestão de carboidratos simples, além de fatores de estresse e influências epigenéticas. 
Pesquisadores têm procurado identificar se aspectos fisiológicos e metabólicos, como o gasto energético em resposta à prática de atividade física, e o consumo calórico decorrente da ingestão de alimentos, estão associados à composição corporal inadequada (DAYRELL et al., 2009; MIRKOPOULOU et al., 2010; ZALILAH et al., 2006).

No entanto, os achados são controversos, alguns autores detectaram que fatores do estilo de vida, como consumo adequado de hortaliças e maior capacidade aeróbia refletiram diretamente em níveis adequados de gordura corporal (DAYRELL et al., 2009). Outros pesquisadores não encontraram nenhuma associação entre consumo alimentar e status do peso corporal (MIRKOPOULOU et al., 2010), demonstrando que outros fatores podem contribuir com a distribuição da gordura no corpo.

A comparação entre 0 gasto energético e 0 consumo de calorias em adolescentes de diferentes níveis de adiposidade corporal foi estudada por MIRKOPOULOU et al.,(2010); e $\underline{\text { ZALILAH }}$ et al.(2006). Zalilah et al. (2006) compararam o consumo calórico e o gasto energético de adolescentes com baixo peso, eutróficos e com excesso de peso e detectaram que meninos com baixo peso apresentaram maior consumo e gasto energético que os demais. Por sua vez, Mirkopoulou et al. (2010) não encontraram nenhuma diferença significativa na ingestão de macronutrientes e no consumo calórico total ao comparar adolescentes de baixo peso com os de sobrepeso/obesidade. Desta forma, a literatura não é conclusiva sobre a relação entre gasto e consumo calóricos nesta faixa etária.

Uma das possíveis razões para tal controvérsia pode estar relacionada ao procedimento empregado para avaliar a composição corporal dos adolescentes. Estas investigações (DAYRELL et al., 2009; MIRKOPOULOU et al., 2010; ZALILAH et al., 2006) empregaram o índice de massa corporal (IMC) na classificação dos grupos em relação à adiposidade corporal. Embora o IMC seja bastante utilizado nesta classificação, alguns autores tecem críticas do seu uso na população de adolescentes, sobretudo entre rapazes. Nesta faixa etária há variação da composição corporal induzida pela ação hormonal, que privilegia o aumento da massa livre de gordura, que por sua vez causa uma classificação equivocada do indivíduo, pois pode não ser distinguida por meio do IMC (FREEDMAN et al., 2007; NEOVIUS et al., 2004).

Assim, a classificação da adiposidade corporal por meio de dobras cutâneas é uma maneira mais precisa que o IMC para a avaliação da composição corporal, por analisar somente a gordura subcutânea (NEOVIUS et al., 2004). Neste sentido, é esperado que estudos em indivíduos com diferentes quantidades de adiposidade, classificados por meio da gordura corporal propriamente dita, poderão detectar variações na influência do gasto e consumo calórico sobre a composição corporal de adolescentes. Com este objetivo, o presente estudo visou comparar o gasto energético e consumo calórico de adolescentes do sexo masculino com diferentes percentuais de gordura corporal.

\section{Métodos}

Esse estudo de delineamento transversal faz parte do projeto intitulado "Relação entre nível de atividade física e hábitos alimentares de adolescentes e estilo de vida dos pais", realizado no ano de 2004 (ILHA, 2004), com adolescentes do sexo masculino da cidade de Florianópolis, Santa Catarina, Brasil. Os protocolos de intervenção no estudo foram aprovados por um Comitê de Ética em Pesquisa com Seres Humanos (Protocolo UFSC, 047/2003).

\section{Sujeitos da pesquisa}

Os sujeitos da pesquisa foram selecionados de forma intencional em uma escola da cidade de Florianópolis, SC, caracterizada como instituição com crianças e adolescentes de alto nível econômico. Os adolescentes deveriam respeitar alguns critérios de inclusão: a) estar na faixa etária de 14 a 16 anos de idade; b) não ser fumante; c) ser de cor branca; d) possuir nível econômico elevado (ASSOCIAÇÃO BRASILEIRA DE EMPRESAS DE PESQUISA, 2000). Tais critérios foram considerados para evitar possível viés de seleção, pois são influenciadores do equilíbrio energético e consequentemente, do acúmulo de peso corporal (NATIONAL ACADEMY OF SCIENCES, INSTITUTE OF MEDICINE, FOOD AND NUTRITION BOARD, 2005). Além desses critérios, os sujeitos deveriam possuir baixo $\% G$, inferior a $11 \%$, ou alto $\% G$, superior a 
$20 \%$, como níveis abaixo e acima do ideal, respectivamente (LOHMAN, 1987).

Após a triagem inicial no estabelecimento de ensino, foram selecionados, de forma aleatória, 40 adolescentes do sexo masculino, divididos em dois grupos: $\% G$ abaixo do ideal $(n=20)$, inferior a $11 \%$; e $\% G$ acima do ideal $(n=20)$, superior a $20 \%$.

O cálculo de tamanho de sujeitos em cada grupo foi realizado a posteriori. Para tanto, empregou-se valores médios e desvio padrão das variáveis do presente estudo. Além disso, foram considerados os seguintes procedimentos: nível de significância de 95\%, poder do teste para comparação de duas médias de 0,80 , razão entre os grupos de 1:1. Além dos valores das variáveis do presente estudo, analisaram-se dados existentes na literatura para verificar se a amostra do presente estudo tinha poder suficiente para garantir a validade interna dos resultados. Os valores médios e o respectivo desvio padrão para as variáveis gasto energético e consumo calórico foram identificados na literatura (ZALILAH et al., 2006), dos grupos de adolescentes com baixo peso e excesso de peso. Após os cálculos, a quantidade necessária de sujeitos para evitar erros do tipo II e garantir validade interna nos resultados da pesquisa seria de, no mínimo, 18 sujeitos em cada grupo.

\section{Instrumentos e procedimentos}

As medidas de massa corporal, estatura e dobras cutâneas do tríceps e subescapular foram mensuradas por um único avaliador treinado, seguindo as padronizações contidas em Petroski (2003). A massa corporal foi medida com uma balança digital da marca Plenna ${ }^{\circledR}$, modelo Suit Prata (São Paulo, Brasil) com capacidade máxima de 150 kg; e a estatura com fita métrica da marca Cardiomed $^{\circledR}$ (Curitiba, Brasil) com resolução de $0,1 \mathrm{~cm}$, fixada verticalmente na parede. A partir da divisão da massa corporal $(\mathrm{kg})$ pelo quadrado da estatura $(\mathrm{m})$, foi calculado o IMC. As espessuras das dobras cutâneas foram aferidas com adipômetro da marca Lange ${ }^{\circledR}$ (Ann Arbor, Estados Unidos) com resolução de $1 \mathrm{~mm}$. Para o cálculo do \%G, somaram-se as dobras cutâneas do tríceps e subescapular, e aplicou-se na equação adaptada por Lohman (1986), que considera a idade, o sexo e a etnia.

O gasto energético foi mensurado por meio de sensor de movimento triaxial, que detecta a aceleração do corpo em três eixos (ânteroposterior, médio-lateral e vertical). $\mathrm{O}$ acelerômetro Tri-axial Research Tracker - TriTrac, modelo RT3, fabricado pela Stayhealthy ${ }^{\circledR}$ (Monrovia, Estados Unidos), foi utilizado por 12 horas diárias, com coleta de dados a cada minuto, em três dias da semana (dois dias durante a semana e um dia durante o final de semana, consecutivos, i.e., domingo, segunda e terça-feira, ou quinta, sextafeira e sábado). Esse aparelho é considerado um método direto para avaliar o dispêndio energético e apresenta indicadores satisfatórios de validade para uso em três dias (WELK; CORBIN, 1995). A estimativa do gasto energético também foi realizada pelo diário de atividades proposto por Bouchard et al. (1983), no qual o adolescente registrava as atividades realizadas a cada 15 minutos. Tal instrumento apresenta reprodutibilidade de $\mathrm{r}=0,91$ em indivíduos a partir de 10 anos de idade (BOUCHARD et al., 1983). Além disso, o diário de Bouchard et al. (1983) mostrou coeficiente de correlação de 0,87 com o acelerômetro TriTrac (WICKEL et al., 2006) e variação intraindividual insignificante em estudo de três dias (WICKEL; EISENMANN, 2006). A partir da estimativa do gasto energético dos dois instrumentos, calculou-se o valor médio para estimar o gasto energético diário.

Optou-se pela avaliação do gasto energético em três dias visando representar as atividades desenvolvidas no ambiente escolar e no tempo livre durante os dias da semana (dois dias) e para representar atividades desenvolvidas no final de semana, seguindo as sugestões de Matsudo et al. (1998) que recomendaram, no mínimo, três dias de monitorização para ter perfil verdadeiro da atividade física habitual. A opção pelos dois instrumentos de medida empregados para avaliar o gasto energético foi devido à impossibilidade logística do emprego do acelerômetro durante o período de 24 horas. Nesse sentido, com o uso do diário foi possível avaliar as demais 12 horas que o sujeito não estava com o acelerômetro.

O consumo calórico foi avaliado pelo consumo energético, estimado por meio de registro alimentar de 24 horas, nos mesmos dias de utilização do acelerômetro. O registro alimentar é considerado um procedimento válido para medir a ingestão alimentar (CAVALCANTE et al., 2004). Os indivíduos foram orientados a anotar os dados de consumo logo após as refeições. Os registros alimentares foram analisados quanto ao valor energético e à distribuição em macronutrientes, 
com o auxílio do software NUT-WIN ${ }^{\circledR}$ (São Paulo, Brasil). Considerou-se 'refeição' todo o momento em que fossem ingeridos alimentos fontes de nutrientes essenciais (LENNERNÄS; ANDERSSON, 1999).

\section{Tratamento estatístico}

O gasto e o consumo energético foram avaliados de forma absoluta (Kcal/dia), e relativa ao peso corporal em quilos por dia (kcal/kg/dia). Para a caracterização das variáveis analisadas, recorreu-se à estatística descritiva (média e desvio padrão). Os dados apresentaram distribuição normal (teste de Shapiro-Wilk), portanto, aplicou-se o teste "t" de Student para amostras independentes ao se comparar os dois grupos. Além disso, foi calculado o desequilíbrio energético pela diferença entre a ingestão e o gasto energético (NATIONAL ACADEMY OF SCIENCES, INSTITUTE OF MEDICINE, FOOD
AND NUTRITION BOARD, 2005), sendo que a comparação intragrupo entre o gasto e a ingestão foi realizada pelo teste " $\mathrm{t}$ " para amostras pareadas. Todas as análises respeitaram um nível de significância 5\%.

\section{Resultados}

Os adolescentes com \%G acima do ideal apresentaram-se mais pesados, com maior IMC e $\% G$ que aqueles com \%G abaixo do ideal $(p<0,05)$, o que era esperado devido aos critérios adotados (Tabela 1).

Verifica-se ainda na Tabela 1, que o gasto energético absoluto semanal foi similar entre os sujeitos com \%G abaixo e acima do ideal. No entanto, os sujeitos com \%G abaixo do ideal tiveram menor consumo calórico absoluto durante toda a semana que os adolescentes com \%G acima do ideal $(p<0,05)$.

Tabela 1. Valores comparativos das características antropométricas, do gasto energético e consumo calórico (kcal.dia ${ }^{-1}$ ) entre adolescentes do sexo masculino com percentual de gordura abaixo e acima do ideal.

\begin{tabular}{lcc}
\hline \multirow{2}{*}{ Variáveis } & \%G abaixo do ideal & \%G acima do ideal \\
\cline { 2 - 3 } & $\bar{X}($ d.p $)$ & $\bar{X}($ d.p $)$ \\
\hline Massa corporal $(\mathrm{kg})$ & $52,4(10,7)$ & $72,6(12,4)^{\star}$ \\
Estatura (cm) & $166,9(9,0)$ & $172,4(10,2)$ \\
IMC $\left(\mathrm{kg} / \mathrm{m}^{2}\right)$ & $18,6(2,1)$ & $24,3(3,0)^{*}$ \\
Percentual de gordura corporal (\%) & $9,1(1,2)$ & $24,6(4,2)^{*}$ \\
Gasto energético diário & & \\
(Kcal/dia) & & $2803,5(524,3)$ \\
$\quad$ Dias da semana & $2672,8(425,6)$ & $2707,6(539,3)$ \\
$\quad$ Final de semana & $2533,8(401,6)$ & $2755,6(531,8)$ \\
$\quad$ Média semanal & $2603,3(413,6)$ & \\
Consumo calórico diário & & $2950,4(520,8)^{*}$ \\
(Kcal/dia) & & $2894,3(806,0)^{*}$ \\
$\quad$ Dias da semana & & $2922,4(663,4)^{*}$ \\
\hline Final de semana
\end{tabular}

$\bar{X}$ - média; d.p - desvio padrão; \%G - percentual de gordura corporal; * $\mathrm{p}<0,05$ (teste "t" de Student para amostras independentes, comparação entre adolescentes: \%G abaixo vs. \%G acima).

Na figura 1A, observa-se que os adolescentes com \%G abaixo do ideal apresentaram gasto energético relativo superior ao consumo calórico durante a semana, o que resultou em um desequilíbrio energético de $-8,3 \mathrm{kcal} / \mathrm{kg} / \mathrm{dia}$ $(p<0,05)$. Nos adolescentes com \%G acima do ideal (Figura 1B), a ingestão calórica superou 0 gasto energético relativo $(p<0,05)$, descrevendo um desequilíbrio no balanço energético relativo, tanto durante a semana $\left(3,5 \quad \mathrm{Kcal} . \mathrm{kg}^{-1} \cdot \mathrm{dia}^{-1}\right)$ quanto no final de semana $\left(2,7 \mathrm{Kcal} \cdot \mathrm{kg}^{-1} \cdot \mathrm{dia}^{-1}\right.$, $p>0,05)$. Ao comparar os dois grupos, foi encontrado que adolescentes com \%G abaixo do ideal apresentaram gasto energético, tanto durante a semana quanto no final de semana, superior àqueles com $\% G$ acima do ideal $(p<0,05)$. 


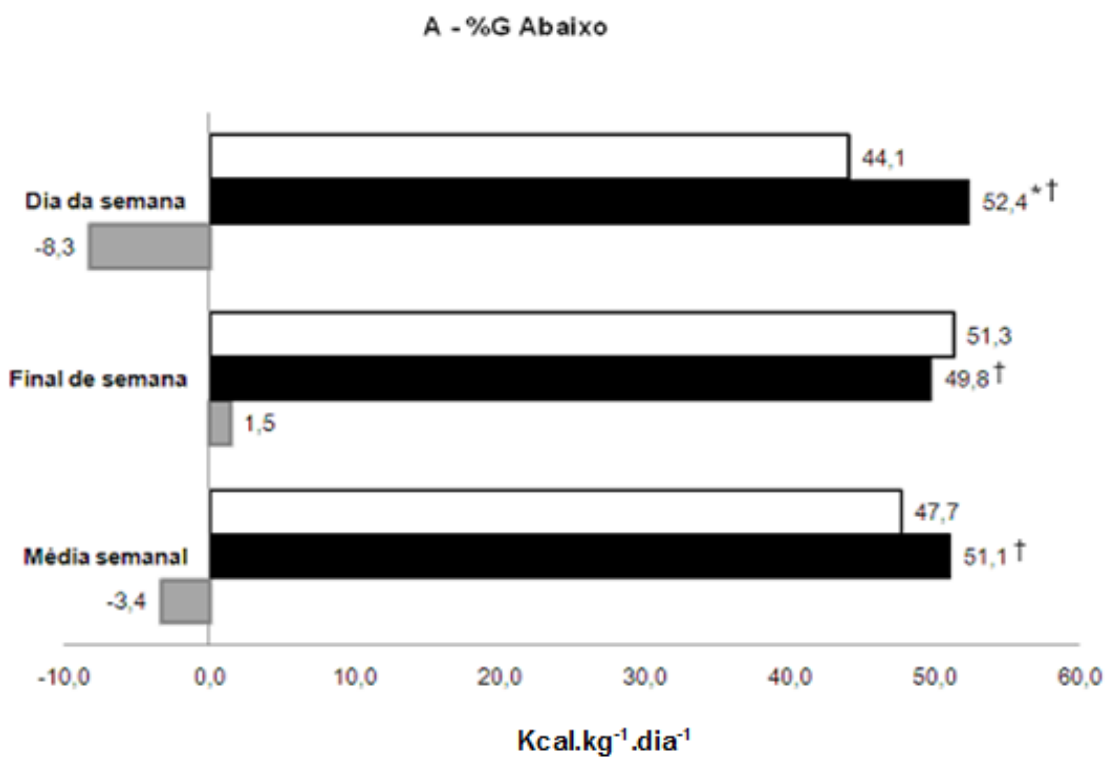

QConsumo energètico $=$ Gasto energètico a Desequilibrio energético

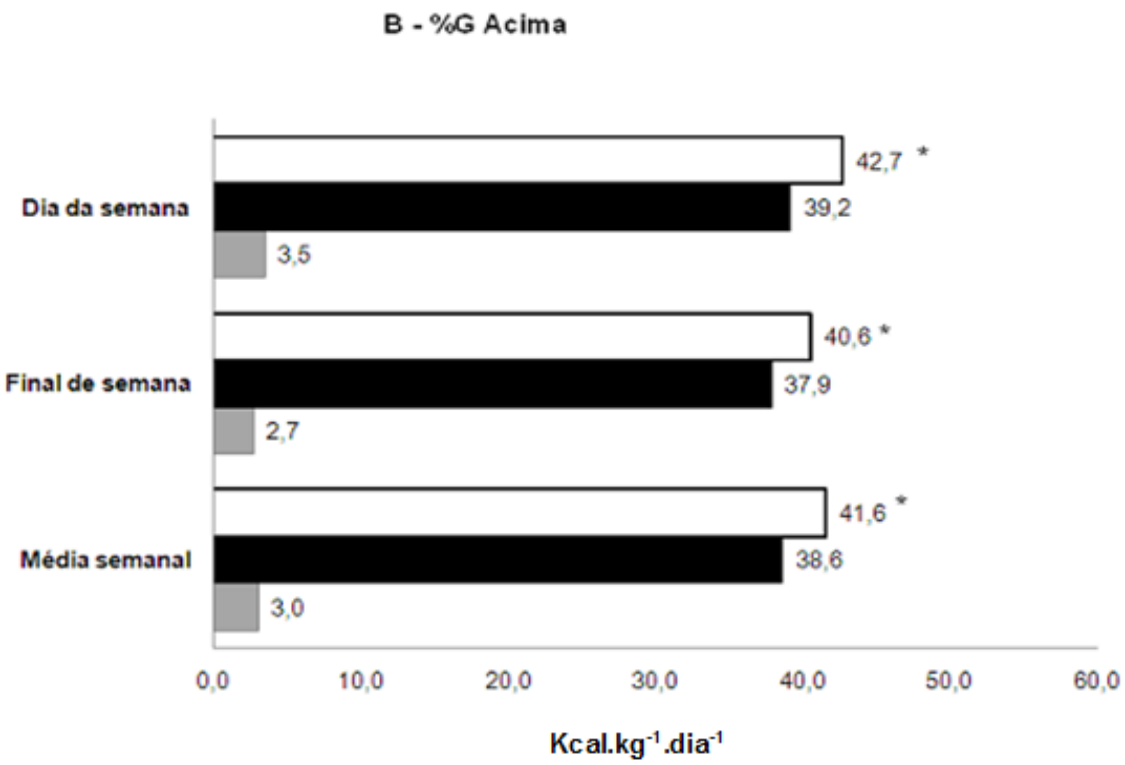

Figura 1. Balanço energético relativo dos adolescentes com $\% \mathrm{G}$ abaixo do ideal (Figura $1 \mathrm{~A}$ ) e com $\% \mathrm{G}$ acima do ideal (Figura 1B). ${ }^{*} \mathrm{p}<0,05$ (teste "t" para amostras pareadas - comparação entre consumo energético e gasto energético intragrupo); $\uparrow \mathrm{p}<0,05$ (teste "t" para amostras independentes - comparação entre os grupos, \%G Abaixo vs. \%G Acima).

\section{Discussão}

O principal achado dessa pesquisa foi que adolescentes com \%G acima do ideal apresentaram menor gasto energético relativo e maior consumo calórico semanal que seus pares com \%G abaixo do ideal, o que sugere ser o estilo de vida um dos principais determinantes da composição corporal dos jovens.
O gasto energético diário compreende o dispêndio energético basal (necessário para a realização das funções vitais do organismo), o gasto energético com atividade física e o efeito térmico dos alimentos (relacionado com a digestão, a absorção e o metabolismo dos alimentos). $O$ gasto energético relacionado à atividade física corresponde, aproximadamente, 
de $15 \%$ a $30 \%$ do gasto diário, e é o mais variável entre indivíduos (NATIONAL ACADEMY OF SCIENCES, INSTITUTE OF MEDICINE, FOOD AND NUTRITION BOARD, 2005). No presente estudo, o gasto energético relacionado à atividade física foi diferente entre os grupos de adolescentes, sendo que os adolescentes com $\% G$ acima do ideal apresentaram um gasto energético relativo inferior, tanto durante a semana quanto no final de semana,

Vasconcelos et al. (2008) verificaram a relação entre gasto energético diário e fatores de risco para doenças cardiovasculares em adolescentes de 12 a 17 anos. Os autores relataram que, independente da quantidade de gordura corporal, adolescentes do sexo masculino com gasto energético diário abaixo de $51,0 \mathrm{Kcal} . \mathrm{kg}^{-1} \cdot \mathrm{dia}^{-1}$ apresentaram maiores concentrações de colesterol total e triglicérides, comparados aos de gasto energético superior. Verifica-se que entre os sujeitos com elevado \%G investigados no presente estudo, esses valores para gasto energético diário foram bem abaixo desta referência, indicando que os adolescentes podem estar expostos a fatores de risco cardiovascular.

A literatura é consistente em relatar que a atividade física e a quantidade de gordura corporal são inversamente proporcionais. Lazzer et al. (2003) compararam o gasto energético em atividades físicas de adolescentes obesos e não obesos. Os jovens com obesidade apresentaram maior gasto energético diário em atividades físicas de intensidade leve, comparados aos não obesos. Por outro lado, os adolescentes não obesos despenderam mais energia em atividades físicas de intensidade moderada e vigorosa. Embora esse estudo tenha avaliado apenas o gasto calórico total, o grupo de indivíduos com baixo \%G apresentou, sistematicamente, maiores valores de gasto em dias de semana e também no final de semana.

As recomendações para consumo calórico diário em adolescentes variam conforme o peso corporal, nível de atividade física e velocidade de crescimento. Em geral, recomenda-se que os jovens consumam, em média, de 2.500 a 3.000 kcal.dia $^{-1}$ (NATIONAL ACADEMY OF SCIENCES, INSTITUTE OF MEDICINE, FOOD AND NUTRITION BOARD, 2005). Os adolescentes do presente estudo com elevada adiposidade corporal, estão atendendo as recomendações da literatura; no entanto, aqueles com baixo \%G estão, em média, aquém das recomendações no período do final de semana. Esse déficit em calorias durante o final de semana não pode ser considerado um dos responsáveis pelo baixo \%G desse grupo, pois ao considerar a média da semana, esses jovens atendem as recomendações.

A análise do balanço energético é recomendada para todas as faixas de idade, podendo indicar o status da composição corporal presente e futura (NATIONAL ACADEMY OF SCIENCES, INSTITUTE OF MEDICINE, FOOD AND NUTRITION BOARD, 2005). Verificou-se que os jovens com adiposidade corporal abaixo da ideal gastaram mais energia do que ingeriram, sobretudo, durante a semana. Em relação aos jovens com elevado \%G, o fator responsável pelo acúmulo de gordura pode ser o balanço energético positivo, pois embora eles atendam às recomendações para consumo, os adolescentes gastaram menos energia do que ingeriram. Berntsen et al. (2009) investigaram adolescentes eutróficos noruegueses e encontraram que, independente da presença de doenças crônicas, o gasto energético durante a semana foi maior que no final de semana. Resultados semelhantes foram encontrados em adolescentes eutróficos brasileiros (DAYRELL et al., 2009; PINHO; PETROSKI, 1999). Não foram encontrados na literatura estudos que verificassem o gasto energético durante a semana e final de semana em jovens com baixo \%G.

Autores de pesquisas com delineamento longitudinal são consistentes em relatar que a adiposidade corporal é dependente do balanço energético, se esse for positivo, a adiposidade aumenta, caso seja negativo, tende a diminuir (PHILLIPS et al., 2003). Desta forma, os resultados dos adolescentes do presente estudo, jovens com elevada adiposidade corporal com maior consumo de calorias do que de gasto energético tanto durante a semana quanto no final de semana, parecem corroborar a tendência dos estudos longitudinais.

Como limitação do presente estudo, destacase o fato de não ter investigado a qualidade da dieta dos adolescentes, tendo em vista que alguns podem estar atingindo as recomendações para ingestão de calorias, mas podem não estar dentro dos padrões para o consumo de micro e macronutrientes. Conforme indicado por Hassapidou et al. (2006), o consumo calórico total 
dos meninos gregos não obesos foi maior que 0 dos obesos, entretanto, ao avaliar a ingestão de micro e macronutrientes ficou evidenciado que os jovens não obesos consumiam mais fibra, amido e ferro que os obesos, que por sua vez, ingeriam mais gorduras.

O presente estudo apresenta como pontos fortes a avaliação da atividade física com instrumento de mensuração direta, o acelerômetro. O consumo calórico foi avaliado por meio de registro alimentar de 24 horas, considerado adequado para estimação dessa medida (CAVALCANTE et al., 2004). Além disso, o presente estudo empregou as medidas de dobras cutâneas para separação dos grupos em relação ao \%G, o que o diferencia em relação a outras pesquisas que empregaram $O$ IMC (DAYRELL et al., 2009; MIRKOPOULOU et al., 2010; ZALILAH et al., 2006).

\section{Conclusão}

Pode-se concluir que adolescentes com diferentes percentuais de gordura corporal apresentam padrão diverso de gasto e consumo energéticos, sendo que sujeitos com \%G acima do ideal consomem mais calorias do que gastam, sendo o inverso verdadeiro para aqueles com baixa adiposidade corporal. Esses resultados sugerem que o estilo de vida exerce um impacto na adiposidade corporal. Desta forma, uma das melhores estratégias para a manutenção da composição corporal em níveis adequados é a participação em intervenções que equilibrem a prática de atividades físicas com o consumo de alimentos.

\section{Referências}

ABEP - Associação Brasileira de Empresas de Pesquisa. Critério de Classificação Econômica Brasil. 2000. Disponível em: http://www.abep.org/novo/Content.aspx?SectionID =35. Acesso em: 13 abr. 2001.

BAKER, J.L.; OLSEN, L.W.; SORENSEN, T.I.A. Childhood Body-Mass Index and the Risk of Coronary Heart Disease in Adulthood. The New England Journal of Medicine, Boston, v. 357, n. 23, p. 2329-2337, 2007. Disponível em: http://www.nejm.org/doi/full/10.1056/NEJMoa0725 15. Acesso em: 01 ago. 2010.

BERNTSEN, S.; CARLSEN, K.C.; ANDERSSEN, S.A.; MOWINCKEL, P.; HAGEBERG, R.; BUESO, A.K.; CARLSEN, K.H. Norwegian adolescents with asthma are physical active and fit. Allergy,
Copenhagen, v. 64, n. 3, p. 421-426, 2009.

Disponível em:

http://www3.interscience.wiley.com/journal/121664 987/abstract. Acesso em: 01 ago. 2010.

BOUCHARD, C.A.; TREMBLAY, C.; LEBLANC, G.; LORTIE, R.; SAVARD, R.; THERIAULT, G.A. Method to assess energy expenditure in children and adults. The American Journal of Clinical Nutrition, Bethesda, v. 37, n. 3, p. 461-467, 1983. Disponível em:

http://www.ajcn.org/cgi/reprint/37/3/461. Acesso em: 01 ago. 2010.

CAVALCANTE, A.A.M.; PRIORE, S.E.; FRANCESCHINI, S.C.C. Food consumption studies: general methodological aspects and its use in the evaluation of children and adolescents aged. Revista Brasileira de Saúde Materno Infantil, Recife, v. 4, n. 3, p. 229-240, 2004. doi: http://dx.doi.org/10.1590/S151938292004000300002 .

DAYRELL, C.; URASAKI, R.; GOULART, R.M.M.; RIBEIRO, S.M.L. Consumo alimentar e gasto energético em adolescentes obesos e eutróficos. Revista Paulista de Pediatria, São Paulo, v. 27, n. 4, p. 374-380, 2009. doi: http://dx.doi.org/10.1590/S0103$\underline{05822009000400005 .}$.

EISENMANN, J.C. Insight into the causes of the recent secular trend in pediatric obesity: Common sense does not always prevail for complex, multifactorial phenotypes. Preventive Medicine, Baltimore, v. 42, n. 5, p. 329-335, 2006. doi: http://dx.doi.org/10.1016/i.ypmed.2006.02.002.

FREEDMAN, D.S.; WANG, J.; OGDEN, C.L.; THORNTON, J.C.; MEI, Z.; PIERSON, R.N.; DIETZ, W.H.; HORLICK, M. The prediction of body fatness by BMI and skinfold thicknesses among children and adolescents. Annals of Human Biology, London, v. 34, n. 2, p. 183-194, 2007. doi:

http://dx.doi.org/10.1080/03014460601116860.

HASSAPIDOU, M.; FOTIADOU, E.; MAGLARA, E.; PAPADOPOULOU, S.K. Energy intake, diet composition, energy expenditure, and body fatness of adolescents in northern Greece. Obesity, Silver Spring, v. 14, n. 5, p. 855-862, 2006. Disponível em:

http://www.ncbi.nlm.nih.gov/pubmed/16855195.

Acesso em: 27 abr. 2011.

ILHA, P.M.V. Relação entre nível de atividade física e hábitos alimentares de adolescentes e estilo de vida dos pais. 2004. 96 f. Dissertação (Mestrado em Educação Física) - Centro de Desportos, Universidade Federal de Santa Catarina, Florianópolis, 2004. 
LAZZER, S.; BOIRIE, Y.; BITAR, A.; MONTAURIER, C.; VERNET, J.; MEYER, M.; VERMOREL, M. Assessment of energy expenditure associated with physical activities in free-living obese and nonobese adolescents. The American Journal of Clinical Nutrition, Bethesda, v. 78, n. 3, p. 471-479, 2003.

Disponível em:

http://www.ajcn.org/cgi/content/full/78/3/471.

Acesso em: 01 ago. 2010.

LEITE, N.; MILANO, G.E.; CIESLAK, F.; LOPES, W.A.; RODACKI, A.; RADOMINSKI, R.B. Effects of physical exercise and nutritional guidance on metabolic syndrome in obese adolescents.

Revista Brasileira de Fisioterapia, São Carlos, v. 13, n. 1, p. 73-81, 2009. Disponível em: http://www.scielo.br/pdf/rbfis/2009nahead/007 09. pdf. Acesso em: 01 ago. 2010.

LENNERNÄS, M.; ANDERSSON, I. Food-based classification of eating episodes (FBCE).

Appetite, London, v. 32, n. 1, p. 53-65, 1999. doi: http://dx.doi.org/10.1006/appe.1998.0196.

LOHMAN, T.G. Applicability of body composition techniques and constants for children and youths. Exercise and Sport Sciences Reviews, New York, v. 14, p. 325-357, 1986. Disponível em: http://www.ncbi.nlm.nih.gov/pubmed/3525188. Acesso em: 01 ago. 2010.

LOHMAN, T.G. The use of skinfold to estimate body fatness on children and youth. Journal of Physical Education, Recreation \& Dance, Reston, v. 58 n. 9 p. 98-102, 1987. Disponível em: http://www.eric.ed.gov/ERICWebPortal/search/det ailmini.jsp? nfpb=true\& \&ERICExtSearch Search Value 0=EJ364412\&ERICExtSearch SearchType $0=$ no\&accno=EJ364412. Acesso em: 01 ago. 2010.

MARTINS, P.A.; SAWAYA, A.L. Evidence for impaired insulin production and higher sensitivity in stunted children living in slums. The British Journal of Nutrition, New York, v. 95, n. 5, p. 996-1001, 2006. doi:

http://dx.doi.org/10.1079/BJN20061754.

MATSUDO, S.M.M.; ARAÚJO, T.L.; MATSUDO, V.K.R.; ANDRADE, D.R.; VALQUER, W. Nível de atividade física em crianças e adolescentes de diferentes regiões de desenvolvimento. Revista Brasileira de Atividade Física e Saúde, Pelotas, v. 3, n. 4, p. 14-26, 1998. Disponível em: http://www.sbafs.org.br/ artigos/206.pdf. Acesso em: 26 abr. 2011.

MIRKOPOULOU, D.; GRAMMATIKOPOULOU, M.G.; GEROTHANASI, K.; TAGKA, A.; STYLIANOU, C.; HASSAPIDOU, M. Metabolic indices, energy and macronutrient intake according to weight status in a rural sample of 17- year-old adolescents. Rural and Remote Health, Geelong, v. 10, n. 4, p. 1513, 2010. Disponível em:

http://www.rrh.org.au/articles/subviewnew.asp?Arti clelD=1513. Acesso em: 26 abr. 2011.

NATIONAL ACADEMY OF SCIENCES, INSTITUTE OF MEDICINE, FOOD AND NUTRITION BOARD. Dietary Reference Intakes for Energy, Carbohydrate, Fiber, Fat, Fatty Acids, Cholesterol, Protein, and Amino Acids (Macronutrients). 2005. Disponível em: http://www.nap.edu/openbook.php?isbn=0309085 373. Acesso em 01 ago. 2010.

NEOVIUS, M.; LINNÉ, Y.; BARKELING, B.; RÖSSNER, S. Discrepancies between classification systems of childhood obesity. Obesity Reviews, Oxford, v. 5, n. 2, p. 105-114, 2004. doi: http://dx.doi.org/10.1111/i.1467789X.2004.00136.x.

PETROSKI, E.L. (org.). Antropometria: técnicas e padronizações. $2^{\mathrm{a}}$ ed. Porto Alegre: Palotti, 2003.

PHILLIPS, S.M.; BANDINI, L.G.; CYR, H.; COLCLOUGH-DOUGLAS, S.; NAUMOVA, E.; MUST, A. Dairy food consumption and body weight and fatness studied longitudinally over the adolescent period. International Journal of Obesity and Related Metabolic Disorders, Hampshire, v. 27, n. 9, p. 1106-1113, 2003. doi: http://dx.doi.org/10.1038/sj.ijo.0802370.

PINHO, R.A.; PETROSKI, E.L. Nível habitual de atividade física e equilíbrio energético em adolescentes. Revista Brasileira de Atividade

Física \& Saúde, Pelotas, v. 4, n. 2, p. 5-16, 1999. Disponível em:

http://www.sbafs.org.br/ artigos/169.pdf. Acesso em: 30 mai. 2010.

SANTOS, C.D.L.; CLEMENTE, A.P.G.; MARTINS, P.A.; SAWAYA, A.L. Influência do déficit de estatura nos desvios nutricionais em adolescentes e pré-adolescentes. Revista de Nutrição, Campinas, v. 22, n. 2, p.187-194, 2009.

Disponível em:

http://www.scielo.br/scielo.php?script=sci arttext\& pid=S1415-52732009000200001. Acesso em: 01 ago. 2010.

VASCONCELOS, I.Q.A.; STABELINI NETO, A.; MASCARENHAS, L.P.G.; BOZZA, R.; ULBRICH, A.Z.; CAMPOS, W.; BERTIN, R.L. Fatores de risco Cardiovascular em Adolescentes com diferentes níveis de gasto energético. Arquivos Brasileiros de Cardiologia, Rio de Janeiro, v. 91, n. 4, p. 227-233, 2008. Disponível em: http://www.scielo.br/pdf/abc/v91n4/04.pdf. Acesso em: 01 ago. 2010. 
WELK, G.J.; CORBIN, C.B. The validity of the

Tritrac-R3D Activity Monitor for the assessment of physical activity in children. Research Quarterly for Exercise and Sport, Washington, v. 66, n. 3, p. 202-209, 1995. Disponível em:

http://www.ncbi.nlm.nih.gov/pubmed/7481081. Acesso em: 27 abr. 2011

WICKEL, E.E.; EISENMANN, J.C. Within- and between-individual variability in estimated energy expenditure and habitual physical activity among young adults. European Journal of Clinical Nutrition, London, v. 60, n. 4, p. 538-544, 2006. doi: http://dx.doi.org/10.1038/sj.ejcn.1602348.

WICKEL, E.E.; WELK, G.J.; EISENMANN, J.C. Concurrent validation of the Bouchard Diary with an accelerometry-based monitor. Medicine and

Science in Sports and Exercise, Madison, v. 38, n. 2, p.373-379, 2006. Disponível em:

http://journals.Iww.com/acsm-

msse/pages/articleviewer.aspx? year $=2006 \&$ issue $=02000$ \&article $=00026 \&$ type $=$ abstract. . Acesso em: 01 ago. 2010.

ZALILAH, M.S.; KHOR, G.L.; MIRNALINI, K.; NORIMAH, A.K.; ANG, M. Dietary intake, physical activity and energy expenditure of Malaysian adolescents. Singapore Medical Journal, Singapore, v. 47, n. 6, p.491-498, 2006.

Disponível em:

http://www.ncbi.nlm.nih.gov/pubmed/16752017. Acesso em: 26 abr. 2011.

\section{Endereço:}

Diego Augusto Santos Silva

Universidade Federal de Santa Catarina

Centros de Desportos/NuCIDH

Campus Universitário - Trindade

Caixa Postal 476

Florianópolis SC Brasil

88040-900

e-mail: diegoaugustoss@yahoo.com.br

Recebido em: 17 de agosto de 2010.

Aceito em: 2 de outubro de 2012.

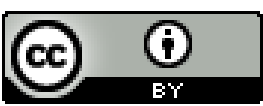

Motriz. Revista de Educação Física. UNESP, Rio Claro, SP, Brasil - elSSN: 1980-6574 - está licenciada sob

Creative Commons - Atribuição 3.0 